\title{
Risk of falling assessment on different types of ground using the instrumented TUG
}

\author{
Mahmoud Ben Brahem \\ Department of Applied \\ Science \\ University of Quebec at \\ Chicoutimi, Canada
}

\author{
Johannes C. Ayena \\ Department of Applied \\ Science \\ UQAC, Canada
}

\author{
Martin J.-D. Otis \\ REPARTI Center, \\ Department of Applied \\ Science \\ UQAC, Canada \\ Martin.Otis@uqac.ca
}

\author{
Bob-Antoine J. Ménélas \\ Department of Mathematics \\ and Computer Sciences, \\ UQAC, Canada
}

\begin{abstract}
Degradation of postural control observed with aging is responsible for balance problems in the elderly, especially during the activity of walking. This gradual loss of performance generates abnormal gait, and therefore increases the risk of falling. This risk worsens in people with neuronal pathologies like Parkinson and Ataxia diseases. Many clinical tests are used for fall assessment such as the Timed up and go (TUG) test. Recently, many works have improved this test by using instrumentation, especially body-worn sensors. However, during the instrumented TUG (iTUG) test, the type of ground can influence risk of falling. In this paper, we present a new methodology for fall risk assessment based on quantitative gait parameters measurement in order to improve instrumented TUG test. The proposed measurement unit is used on different types of ground, which is known to affect human gait. The methodology is closer to the real walking environment and therefore enhances ability to differentiate risks level. Our system assesses the risk of falling's level by quantitative measurement of intrinsic gait parameters using fuzzy logic. $\mathrm{He}$ is also able to measure environmental parameters such as temperature, humidity and atmospheric pressure for a better evaluation of the risk in activities of daily living (ADL).
\end{abstract}

Keywords: Risk of falling; gait analysis; instrumented TUG; Type of ground

\section{INTRODUCTION}

Falls are partly consequence of the age-related gait disorders. Frequently, the occurrence of falls in the elderly causes physical and psychological damage with considerable costs $[1,2]$, which makes it a concern of public health. Falls are often the result of an interaction between extrinsic factors related to the physical environment (soil types, climatic conditions, etc.) and intrinsic factors associated to the health of the individual (visual deficiency, decreased mobility of the lower limbs, proprioceptive degradation and cognitive impairment, etc.) [3]. This observation leads us to study these different factors in order to evaluate a risk of falling in clinical test and then propose enhance diagnostic aid for instrumented Timed Up and Go (iTUG). Our suggested system presented in this paper should be used in order to calibrate the algorithm for each person in a controlled environment. Then, this system could be used in activities of daily living (ADL).
The Timed Up and Go (TUG) test is one of the multiple tests enabling analysis of balance problems; other examples are the Tinetti test [4] and Sit-to-Stand test (STST) [5]. During the TUG test [6], the participant gets up from a chair, walks straight for three meters, turns to return to the seat and sit down. Many previous researches have focused on the TUG test. The first criterion to assess the risk of falling is the test duration. The risk of falling is considered higher when the person exceeds the normal average duration of the test $[7,8]$. The efficiency of the TUG in predicting falls and distinguishing fallers from non-fallers is approved by many geriatrics societies in the world. However, it is noted that this test is based on coarse and subjective criterions [2]. For example, it does not consider the variability of temporal gait parameters while walking in straight line, which affects directly the risk of falling's. Thus, the duration of the TUG test does not appear sufficient to estimate this risk. Many researchers have suggested enhancing the test by using bodyworn sensors without compromising its simplicity [9-11].

This paper proposes a new methodology for assessing risk of fall using a wearable low-cost system. A diagnostic aid for the fall risk could be improved using iTUG combined to realistic environment. Therefore, this new system is evaluated on different types of ground in order to better reproduce daily situations since the ground is one of several disturbances that have an impact on gait parameters [12]. The IMU signals allow us to estimate the variability of the intrinsic parameters such as stride length variability, cadence, step length, the swinging time of foot, etc. The proposed wearable system is also able to measure environmental parameters like temperature, humidity and atmospheric pressure. Those extrinsic parameters represent additional perturbations affecting the risk of falling.

The structure of the paper is organized into the following sections. First, a related work on the gait analysis using instrumented TUG is presented in order to demonstrate the main contribution of this paper. Second, the conception of the proposed wearable system and the used methodology for 
assessing risk of falling are detailed. Third, a risk of falling is evaluated with some experiments using iTUG.

\section{RELATED WORK}

Many studies on gait analysis of elderly people were published. They helped clarify some gait parameters as they appear specifically modified or disrupted in the elderly. To study human gait parameters, some authors use various devices and method for evaluating gait parameters such as cadence and step length. These experiences have similarities and differences, and most of them use inertial measurement unit (IMU) located at various locations on the body to acquire data related to limb motion [13-15]. This section review research works related to both aspects for risk level assessment: the first one concerns the wearable devices whereas the second is about using an instrumented shoe for gait improvement in TUG test.

Ohtaki et al [16] used a device attached to the leg for detecting temporal gait parameters and calculating the bending angle of the ankle. The equipment used contains a pair of onedimensional accelerometer and gyroscope. Despite the efficiency of their methodology, they use a complex instrumental device that could not be used or worn by the elderly. Other researchers [17] evaluate gait parameters with devices attached to the belt. An alternative solution is the design of a device attached to the shoe. They are several device models fixed to the side [18], forward [19], or backward [20,21]. The most are equipped with resistive force sensors for detecting phases of gait such as the instrumented sole presented in [22]. However, such sensors limit the autonomy of the device and thus its mobility.

To increase the usefulness of the TUG, several studies used an instrumented approach, especially body-worn sensors [23, 24]. Barry and al [25] exploited two SHIMMER kinematic sensors to assess fall risk using the items of the TUG tests. Each kinematic sensor contains both a triaxial accelerometer and a triaxial gyroscope that were attached to the anterior of each shank and trunk. They reported 44 gait parameters which 29 provided significant discrimination between participants as fallers and non-fallers.

Parkinson's disease (PD) is one of the most prevalent neurodegenerative diseases. Most people with PD have balance problems which worsen with disease progressive [26]. Symptoms of this disease, in particular motors symptoms such as festination, freezing, etc. increase risk of falling as compared to healthy individuals at the same age, but also in relation to people with other neurological disorders [27]. In order to detect abnormalities in early (PD) Zampieri et al [28] used body-worn sensors for mobility testing at home versus a laboratory testing situation. They used five inertial sensors, which were attached to the dorsum of each wrist, to the anterior shank and to the chest on the sternum. Their results show that home testing is feasible. However, their experiments were conducted at houses with flooring and analysis gait in laboratory was a linoleum floor. In the same idea of flooring usage, Salarian et al [29] also combine seven inertial sensors attached on the forearms, shanks, thighs and sternum for detecting a significant difference in cadence, angular velocity of arm-swing, turning duration and time to perform turn-to-sits in early PD and control subjects. Although many parameters of gait were assessed in their various studies, they don't consider the influence of type of ground and the variability of temporal gait parameters, which affect directly the risk of falling's level. To overcome this situation, Sprager et al [30] used an accelerometer on different surfaces such as ground, glass, gravel and stone plates. For determining gait characteristics, they asked each participant to walk across the surface with their normal pace. Other studies such as presented in $[12,31,32]$ have also demonstrated the influence of surface.

\section{PROPOSED HARDWARE FOR ITUG}

The materiel used in this study is composed of 1) the new wearable measurements system; 2) the walking experimental floor and 3) the motion capture cameras system. The next sections will present the setup used for the proposed iTUG.

\section{A. Environment Setup}

The experimental floor contains five corridors of one meter broad and five meters lengths; each corridor holds different types of ground (sand, gravel, concrete, parquet floor and carpet). The 3D motion capture system is an Optitrack from Natural Point; it uses eight infrareds cameras V100:R2 model, four on each side of the corridor of walking to allow the motion detection. These are cameras with $46^{\circ}$ horizontal and $35^{\circ}$ vertical field of view, .03 MP resolution, frame rate of 100 FPS, sub-millimeter accuracy, latency of $10 \mathrm{~ms}$ and 26 IR LEDs. Reflective markers are located on specifics positions on the leg and the foot, (shown in Fig. 1). During the experiment, the motion capture system allows to follow foot trajectory. They are used as a way of comparison between gait phases seen by the cameras and those detected by our wearable measurement device.
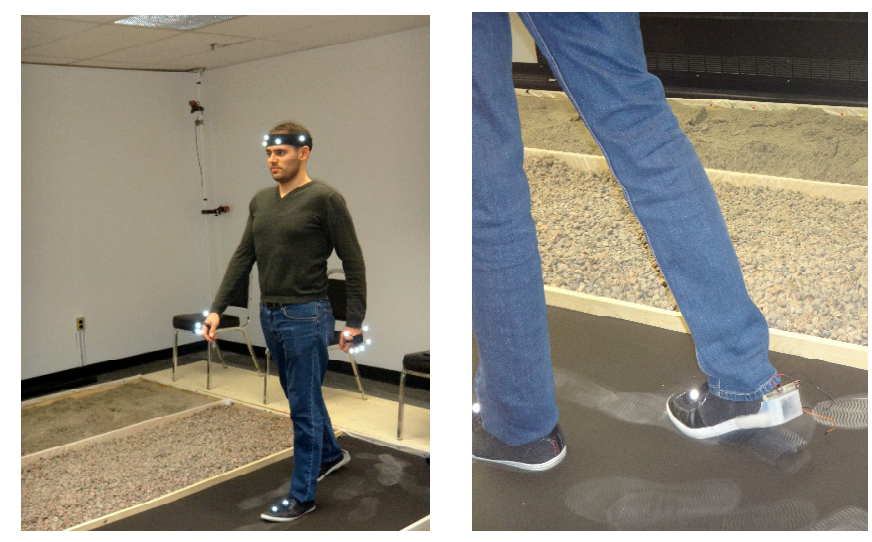

Figure 1. Markers and wearable device positions 


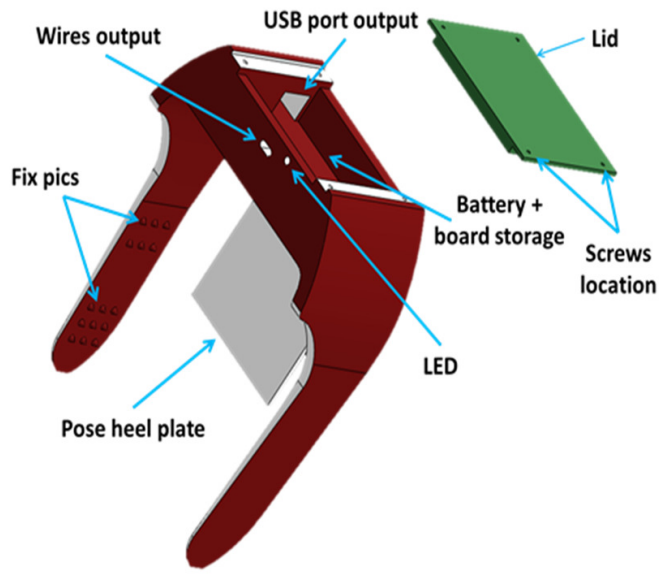

Figure 2. Mechanical enclosure design

\section{B. Instrumentation: Proposed Wearable System}

The mechanical enclosure is designed to be fixed to the rear of the foot, more specifically to the heel of the shoe. This new wearable device, shown in Fig. 2, measures the variability of cadence, stride length and the swinging time of the foot while the person is walking and can differentiate ground physical properties (such as compliance). To satisfy this functionality, a sufficiently flexible material was chosen to adapt our device to most standard sizes shoes, forms and models (for men, women and children). The mechanical enclosure is composed from two pieces; the principal one has a $U$ form to be attachable to the heel of the shoe and be adapted to the majority of standard forms and sizes of shoes as show in Fig. 2. The second mechanical part is a lid to protect the electronic board placed in the first one. The designed enclosure has to be adaptable to most models of standard shoes, receive and protect electronic board, as non-invasive as possible, easily attachable to the shoe being non-destructive. The design was done using SolidWorks software and the prototype was 3D printed.

The selected fabrication material is DSM Somos 9120. This polypropylene prototype material exhibits properties very close to actual polypropylene. Its flexibility makes it an ideal choice for a prototype with pressure fitting or made from assembled parts. This material is also useful in creating parts for applications where durability and robustness are key requirements (such as automotive components, electronic enclosures, medical products, large panels and interlocking parts). These two characteristics are required in our case because the enclosure needs to protect the electronic board while the user is walking on different types of soil.

As regards the electronic part, the circuit is designed to be as small as possible. It provides direct measurement of some parameters such as temperature, humidity, etc. and indirect measurement of other parameters that will be, for example, computed from the acceleration signals (steps cadence, stride length, swing time, etc.). Also, ground vibration is measured from acceleration signal (impulse response of the heel strike) in order to evaluate its compliance. All these data are collected thanks to many sensors installed on the circuit (3-axis accelerometer, 3-axis gyroscope, barometer, 3-axis magnetometer, thermometer and humidity sensor). Furthermore, computation and data analysis are performed by embedded software included in a microprocessor. The board contains also a Micro SD card for the data storage with a Bluetooth for the data transfer to other wireless devices (such as SmartPhone, tablet, etc.). The whole is powered by lithium battery $(400 \mathrm{mAh})$. Sensors and electronic components are presented in table 1.

TABLE I. ELECTRICAL COMPONENTS

\begin{tabular}{|c|l|}
\hline Index & \multicolumn{1}{|c|}{ Description } \\
\hline 1 & 2-axis gyro sensor \\
\hline 2 & Barometric sensor \\
\hline 3 & Management module Lithium \\
\hline 4 & Pulsed voltage regulator \\
\hline 5 & Microcontroller \\
\hline 6 & 3-axis magnetometer sensor / Temperature \\
\hline 7 & Receptacle Micro SD Card \\
\hline 8 & Micro USB receptacle \\
\hline 9 & Gyroscopic Sensor 1 axis \\
\hline 10 & Humidity Sensor \\
\hline 11 & Bluetooth Module \\
\hline 12 & 3-axis accelerometer sensor \\
\hline
\end{tabular}

\section{METHODOLOGY}

In order to estimate the risk of falling during iTUG, we start by analyzing the gait parameters of 12 healthy young participants. Then we used fuzzy logic to estimate the value of risk on each type of ground. The experiment that allowed us to estimate the risk of falling was also presented. The following sub-sections describe each step.

1) Gait parameters analysis: Most of gait parameters have not the same normal range of values for each person. They change according to the gender, age and height to name a few. That's why it is more interesting to detect the temporal variation of those parameters. This variability is calculated using the mean normal value of each participant, which is estimated with a calibration of its normal gait measurements. The measure of stride length and swing time variability allowed us to identify abnormalities in the rhythm of gait. Each parameter variability are calculated versus the mean value of each parameter for each gait cycle. It is considered like abnormal when it exceeds $\pm 10 \%$ [33].

2) Fuzzy logic model: This algorithm is introduced as a standard tool for dealing with complex and non-linear systems. It has the advantage we can define and know how it works and do computation, unlike the other conventional techniques like neural network. Counter the true or false in Boolean algebra, fuzzy logic techniques is based on degrees of truth usage to evaluate risk of falling levels. It also has the advantage of being easier and cheaper to implement than other methods. The library used to implement fuzzy logic in this study is jFuzzyLogic and it is one of the most comprehensive libraries in JAVA language. The principle of a fuzzy system is to compute the output parameters based on a set of rules 
formulated in natural language like the follow examples implemented in our system:

- RULE 22: IF ((cadence IS average) AND (swing_time_var IS average)) AND (stride_length_var IS low) THEN risk IS low;

- RULE 45: IF ((cadence IS very_fast) AND (swing_time_var IS high)) AND (stride_length_var IS high) THEN risk IS high;

These rules provide the corresponding risk of falling based on membership functions and the magnitude of each gait parameters.

The numerical raw data acquired from the sensors are processed in order to compute the variability of gait parameters such as done in [34]. This variability represents the input of fuzzy logic algorithm and specifically its first step, which is the fuzzification. The fuzzification consists in the translation of digital data coming from sensors into fuzzy inputs. The number of inputs corresponds to the fuzzy inputs (gait parameters measured in our study), which describes the intrinsic risk factors. With a membership function set used, as shown in Fig. 3 with the example of gait cadence, quantitative data from acceleration sensor is converted in qualitative linguistic variable. For example, from a certain swing time variation, the algorithm is able to know if it is considered like high or low variation compared to the average values measured on concrete ground. The second step is the elaboration of the inference engine, which consists in the definition of the inference rules. The algorithm's output represents the risk assessment level, which is expressed as low, moderate or high-risk level as suggested in standard TUG. Thereafter, the fuzzy outputs have been determined. The third and final step is the defuzzification, which transforms these variables in numerical outputs. Defuzzification procedures are used to select an adequate decision among those deemed adequate using the output possibility distribution. Two main methods of defuzzification such as the center of gravity (COG) method and the average of maximums (MM) method were used for computing the risk of falling. After testing the outputs of the two methods, the COG method has given better results such that the risks of falling levels can be easily differentiable. The membership functions used in this study for gait parameters measured are showed in Fig. 4, 5 and 6. The membership intervals were determined from the average of gait parameters on concrete walking and their corresponding tolerance intervals.

3) Experimental process: This experiment is based on the classical TUG test using our wearable system. Each participant was walking on different types of ground while motion capture was recording the foot trajectory. The experiment starts when the participant gets up from the chair, walks three (3) meters, turn around and come back to sit in the chair again. The same process is done on each type of ground four times. The duration is recorded like in classical TUG test while our system records gait parameters related to a risk of fall. Twelve healthy subjects did the experiment (18 and 27 years old). They are graduated students and none of them had any gait abnormalities. All subjects were informed about the experiments protocol and gave written consent before participating.

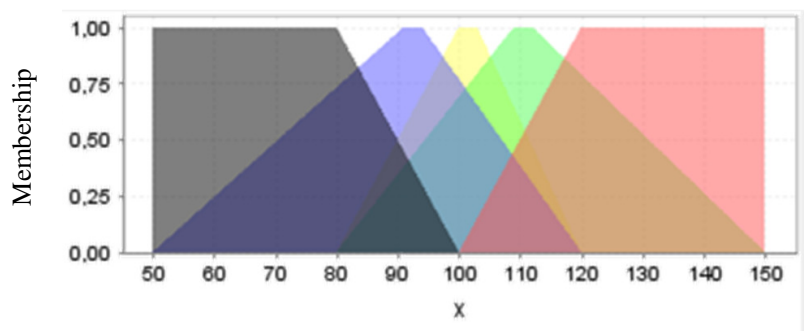

a very_slow a very_fast a slow a fast average

Figure 3. Cadence's membership function

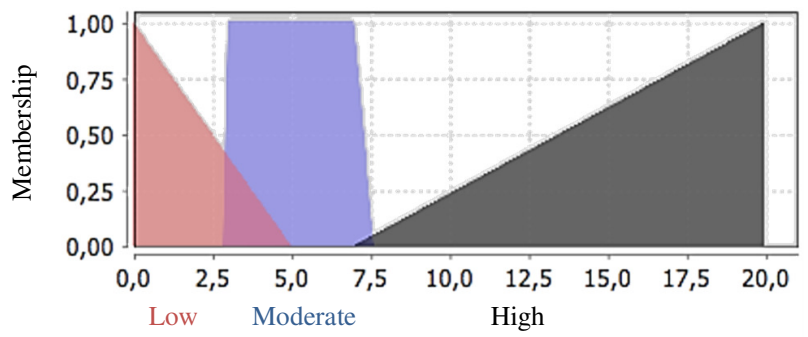

Figure 4. Stance of foot variability

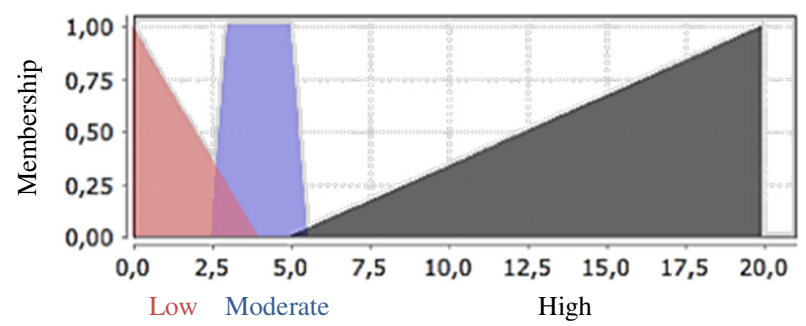

Figure 5. Swing of foot variability
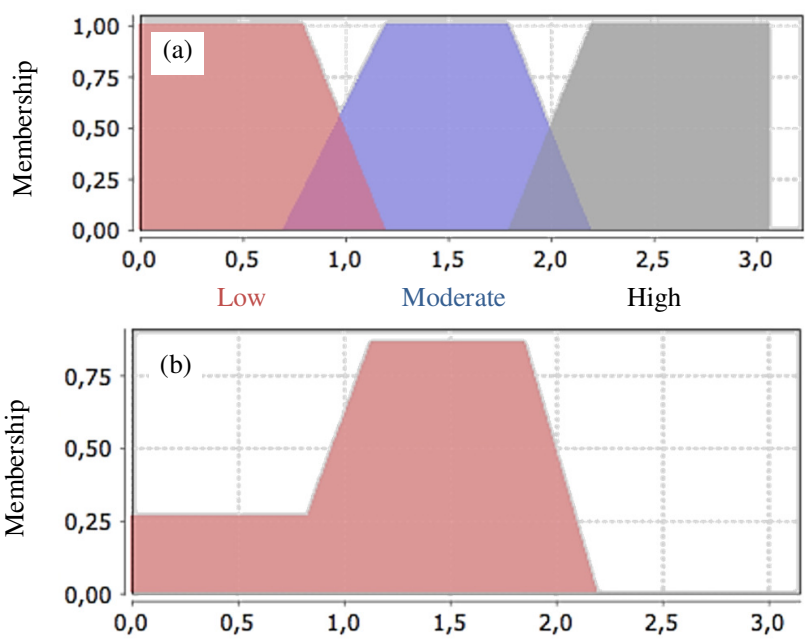

Figure 6. Risk of falling level: (a) membership function used for different risk of falling level and (b) output of the proposed algorithm

\section{RESULTS}

Fig. 8 shows the variability of gait parameters for one of the participants. This variability should come from the impact of walking over the types of ground and then change TUG 
duration as shown in Fig. 7 for six participants. As noted previously, the gait parameters variability have been used for the proposed fuzzy logic algorithm. The output's algorithm represents the risk of falling of each type of ground which can be between 1 to 3 levels (Fig. 9) as suggested in the TUG (level 3 is the highest risk). Moreover, the time necessary to perform the TUG test over each type of ground is recorded for all participants and an ANOVA analysis is presented in Fig. 10.

\section{DISCUSSION}

The purpose of the present study was to propose a new low-cost system for assessing risk of falling at home. The first step is the evaluation of a calibration methodology of the fuzzy logic rules for each person in a controlled environment. In order to take into account real condition at home, various types of ground are used. Despite the healthy young population involved in this study, the results suggest that the type of ground did affect variability of the gait parameters (shown in Fig. 8) and therefore the risk of falling level (shown in Fig. 9). The variability of gait parameters is more pronounced over sand and gravel than concrete, parquet and carpet. This could be explained by the fact that concrete, parquet and carpet are rigid compared to the two others types of ground which require more muscle activities in order to correct foot rotation. Then, the risk of falling is higher over sand and gravel. However, this result is not statistically significant inside the group of participants, but is coherent for each participant. In many cases, young participants showed a small decline in performance over sand, carpet, gravel, parquet compared to concrete probably because muscle need more energy for compensate to compliant ground.

Our findings were consistent with previous works as far as concerned the TUG time in dual task. The time necessary to perform the TUG test is higher over sand than concrete (shown in Fig. 7). Most of participants had difficulty to walk over the sand as suggested in both Fig. 9 and 10.

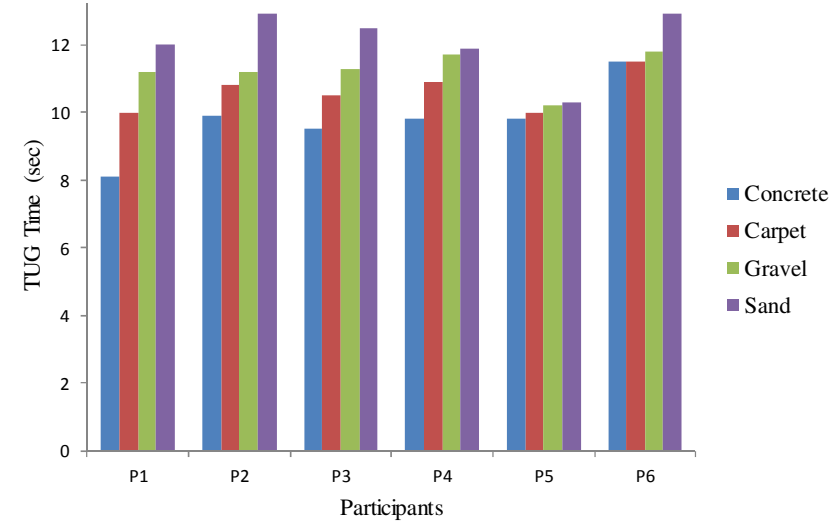

Figure 7. TUG duration performed by six participants

One way Analysis of Variance (ANOVA) was performed in order to compare the TUG duration performed by all participants over concrete, carpet, sand and gravel.

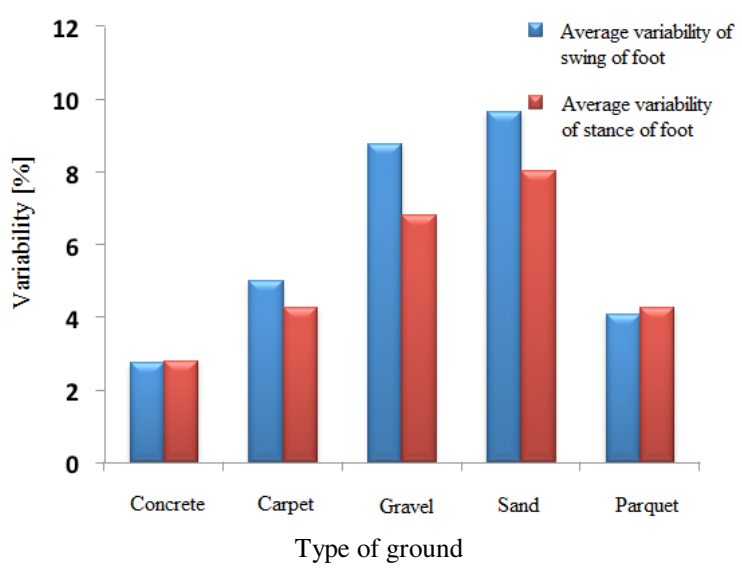

Figure 8. Variability of gait parameters for one of participants

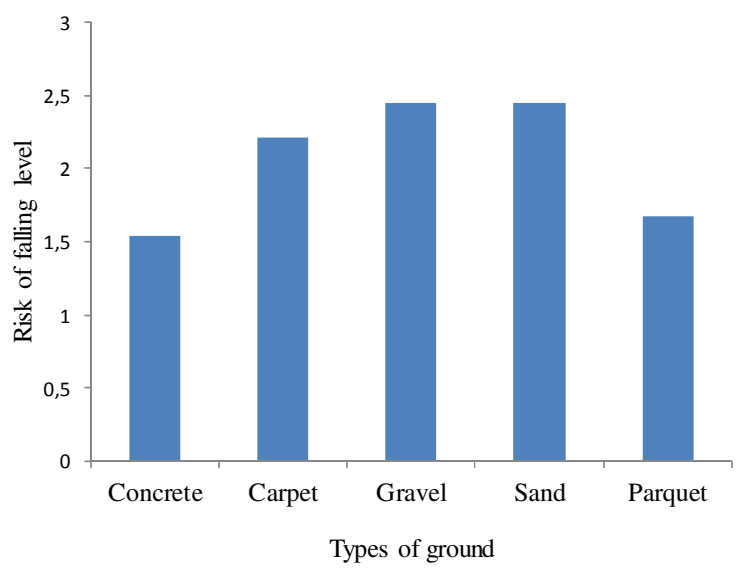

Figure 9. Risk of falling level for all participants over five type of ground

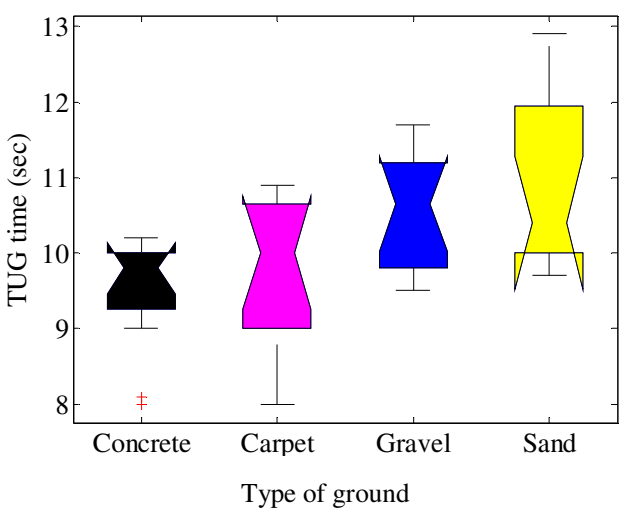

Figure 10. TUG time performed with all participants over four types of ground

The ANOVA result is reported as an F-statistic with its associated degrees of freedom and p-value. The null hypothesis $H_{0}$ is that all the means of TUG duration from different ground are equals. Given that the null hypothesis is rejected if $F>F_{\text {critical }}$ at the 0.05 level of significance, the computed test statistic $F$ in our work is $\mathrm{F}=6.41$ which is more than $F_{\text {critical }}=2.82$. This analysis of variance, in Fig. 10, leads to the conclusion that there has a significant effect related to the types of ground and TUG duration $(\mathrm{F}(3.44)=6.41, p=$ $0.0011<0.05)$. 


\section{CONCLUSION}

In this study, we proposed a new low-cost system for assessing the risk of falling in an instrumented TUG. We also evaluated the effects of four types of ground on the participant's performance during this clinical test. Our results show that fuzzy logic can assess the risk of falling's level by using quantitative measurement of intrinsic gait parameters. Also, a calibration of the fuzzy logic should be done in a controlled environment in order to improve the risk

\section{REFERENCES}

[1] B. Caby, S. Kieffer, M. de Saint Hubert, G. Cremer, and B. Macq, "Feature extraction and selection for objective gait analysis and fall risk assessment by accelerometry," BioMedical Eng OnLine, vol. 10, p. 1, 2011.

[2] J. Howcroft, J. Kofman, and E. Lemaire, "Review of fall risk assessment in geriatric populations using inertial sensors," $J$. of NeuroEngineering and Rehabilitation, vol. 10, p. 91, 2013.

[3] M.-L. Gaubert-Dahan, A. Cougnaud-Petit, L. De Decker, C. Annweiler, O. Beauchet, and G. Berrut, "Beyond to pattern of risk factors in elderly subjects," Ger Psychol Neuropsychiatr Vieil vol. 9 (3), pp. 27785,2011

[4] Tinetti M. E., Baker D.I., McAvay G., Claus E.B., Garrett P., Gottschalk M., et al., "A multifactorial intervention to reduce the risk of falling among elderly people living in the community," N Engl J Med, vol. 331, pp. 821-827, 1994

[5] S. Whitney, L. Wrisley, D. M. Marchetti, G. F. Gee, M. A. Redfern, M. S. Furman, et al., "Clinical measurement of sit-to-stand performance in people with balance disorders: Validity of data for the five-time -sit-tostand test " Physical Therapy vol. 85(10), pp. 1034 - 1045, 2005.

[6] Mathias S., Nayak USL, and I. B., "Balance in elderly patients: the Get Up and Go Test," Arch Phys Med Rehabil, vol. 67, pp. 387-389, 1986.

[7] A. Shumway-Cook, S. Brauer, and M. Woollacott, "Predicting the probability for falls in community-dwelling older adults using the Timed Up \& Go Test," Phys Ther, vol. 80(9), pp. 896-903, 2000 Sept.

[8] D. Podsiadlo and S. Richardson, "The timed "up and go": a test of basic functional mobility for frail elderly persons," J Am Geriatr Soc, vol. 39, pp. 142-148, 1991

[9] N. Bidargaddi, A. Sarela, J. Boyle, V. Cheung, M. Karunanithi, L. Klingbei, et al., "Wavelet based approach for posture transition estimation using a waist worn accelerometer," in IEEE EMBS, 2007, pp. 1884-1887. [10] A. Weiss, T. Herman, M. Plotnik, M. Brozgol, I. Maidan, N. Giladi, et al., "Can an accelerometer enhance the utility of the Timed Up \& Go Test when evaluating patients with Parkinson's disease?," Medical engineering \& physics, vol. 32, pp. 119-125, 2010.

[11] W. G. M. Janssen, B. J. Bussmann, H. L. D. Horemans, and H. J. Stam, "Validity of accelerometry in assessing the duration of the sit-tostand movement," Medical \& Bio Eng. \& Com., vol. 46, pp. 879-887, 2008. [12] D. S. Marigold and A. E. Patla, "Adapting locomotion to different surface compliances: neuromuscular responses and changes in movement dynamics," Journal of neurophysiology, vol. 94, pp. 1733-1750, 2005.

[13] S. M. M. De Rossi, S. Crea, M. Donati, P. Rebersek, D. Novak, N. Vitiello, et al., "Gait segmentation using bipedal foot pressure patterns," in IEEE Biomedical Robotics and Biomechatronics, 2012 June, pp. 361-366.

[14] R. Feliz Alonso, E. Zalama Casanova, and J. Gómez GarcíaBermejo, "Pedestrian tracking using inertial sensors," vol. 3 (1), 2009.

[15] T. Lina, S. Quanjun, G. Yunjian, and L. Ming, "HMM-Based Human Fall Detection and Prediction Method Using Tri-Axial Accelerometer," Sensors Journal, IEEE, vol. 13, pp. 1849-1856, 2013.

[16] Y. Ohtaki, K. Sagawa, and H. Inooka, "A Method for Gait Analysis in a Daily Living Environment by Body-Mounted Instruments," JSME Inter. J. Series C Mechanical Systems, Machine Elements and Manufacturing, vol. 44, pp. 1125-1132, 2001.

[17] D. Tadic, M. Djapan, M. Misita, M. Stefanovic, and D. D. Milanovic, "A fuzzy model for assessing risk of occupational safety in the processing industry," International journal of occupational safety and ergonomics: JOSE, vol. 18, pp. 115-126, 2012.

[18] J. Klucken, J. Barth, P. Kugler, J. Schlachetzki, T. Henze, F. Marxreiter, et al., "Unbiased and Mobile Gait Analysis Detects Motor Impairment in Parkinson's Disease," PLoS ONE, vol. 8, p. e56956, 2013. computation. This paper then suggested all the apparatus in order to complete a calibration for each person in a clinic environment. After this calibration, our wearable device could be used in activities of daily living (ADL) for monitoring risk of fall considering many parameters such as: effect of medication (type, dose, strength, etc.) and disease progression (such as Parkinson, Ataxia, etc.). All those information coming from our system could be submitted to an electronic medical record for diagnostic aid.

[19] D. Hamacher, N. Singh, J. Van Dieën, M. Heller, and W. Taylor, "Kinematic measures for assessing gait stability in elderly individuals: a systematic review," J Royal Society Interface, vol. 8, pp. 1682-1698, 2011.

[20] B. Mariani, C. Hoskovec, S. Rochat, C. Büla, J. Penders, and K. Aminian, "3D gait assessment in young and elderly subjects using footworn inertial sensors," J. of Biomechanics, vol. 43, pp. 2999-3006, 2010.

[21] S. J. M. Bamberg, A. Y. Benbasat, D. M. Scarborough, D. E. Krebs, and J. A. Paradiso, "Gait Analysis Using a Shoe-Integrated Wireless Sensor System," IEEE Tr. Information Tech. in Biomed., vol. 12, pp. 413-423, 2008.

[22] D. Gagnon, B. A. J. Menelas, and M. J. D. Otis, "Qualitative Risk of Falling Assessment Based on Gait Abnormalities," in IEEE Systems, Man, and Cybernetics (SMC), 2013 Oct, pp. 3966-3971.

[23] Y. Higashi, K. Yamakoshi, T. Fujimoto, M. Sekine, and T. Tamura, "Quantitative evaluation of movement using the timed up-and-go test," IEEE Eng. in Medicine and Biology Magazine, vol. 27, pp. 38-46, 2008.

[24] T. Frenken, M. Brell, M. Gövercin, S. Wegel, and A. Hein, "aTUG: technical apparatus for gait and balance analysis within component-based Timed Up \& Go using mutual ambient sensors," Journal of Ambient Intelligence and Humanized Computing, vol. 4, pp. 759-778, 2013.

[25] R. Barry, O. D. Alan, R.-O. Roman, C. Lisa, N. S. Cliodhna, and A. K. Rose, "Quantitative Falls Risk Assessment Using the Timed Up and Go Test," IEEE Tr. on biomedical Eng., vol. 57 (12), pp. 2918-2926, 2010.

[26] S. Deandrea, E. Lucenteforte, F. Bravi, R. Foschi, C. La Vecchia, and E. Negri, "Risk factors for falls in community-dwelling older people: a systematic review and meta-analysis," Epidemiology., vol. 21(5), pp. 65868., 2010 Sep.

[27] B. R. Bloem, Y. A. Grimbergen, M. Cramer, M. Willemsen, and A. H. Zwinderman, "Prospective assessment of falls in Parkinson's disease.," $J$ Neurol, vol. 248(11), pp. 950-958, 2001 Nov.

[28] Z. Cris, S. Arash, C.-K. Patricia, G. John, and B. H. Fay, "Assessing mobility at home in people with early Parkinson's disease using an instrumented Timed Up and Go test," Parkinsonism and Related Disorders vol. 17 pp. 277-280, 2011.

[29] A. Salarian, F. B. Horak, C. Zampieri, P. Carlson-Kuhta, J. G. Nutt, and K. Aminian, "iTUG, a Sensitive and Reliable Measure of Mobility," IEEE Tr. Neural Sys and Rehabilitation Eng., vol. 18, pp. 303-310, 2010.

[30] S. Sprager and D. Zazula, "Impact of different walking surfaces on gait identification based on higher-order statistics of accelerometer data," in IEEE Signal and Image Processing Applications, 2011 Nov, pp. 360-365.

[31] M. Muaaz and C. Nickel, "Influence of different walking speeds and surfaces on accelerometer-based biometric gait recognition," in Telecommunications and Signal Processing, 2012 July, pp. 508-512.

[32] R. Cham and M. S. Redfern, "Changes in gait when anticipating slippery floors," Gait \& posture, vol. 15, pp. 159-171, 2002.

[33] J. Verghese, R. Holtzer, R. B. Lipton, and C. Wang, "Quantitative Gait Markers and Incident Fall Risk in Older Adults," J. Gerontology Series A: Biological Sci. and Medical Sci., vol. 64A, pp. 896-901, 2009.

[34] M. B. Brahem, B.-A. J. Ménélas, and M. J. D. Otis, "Use of a 3DOF Accelerometer for Foot Tracking and Gesture Recognition in Mobile HCI," Procedia Computer Science, vol. 19, pp. 453-460, 2013. 